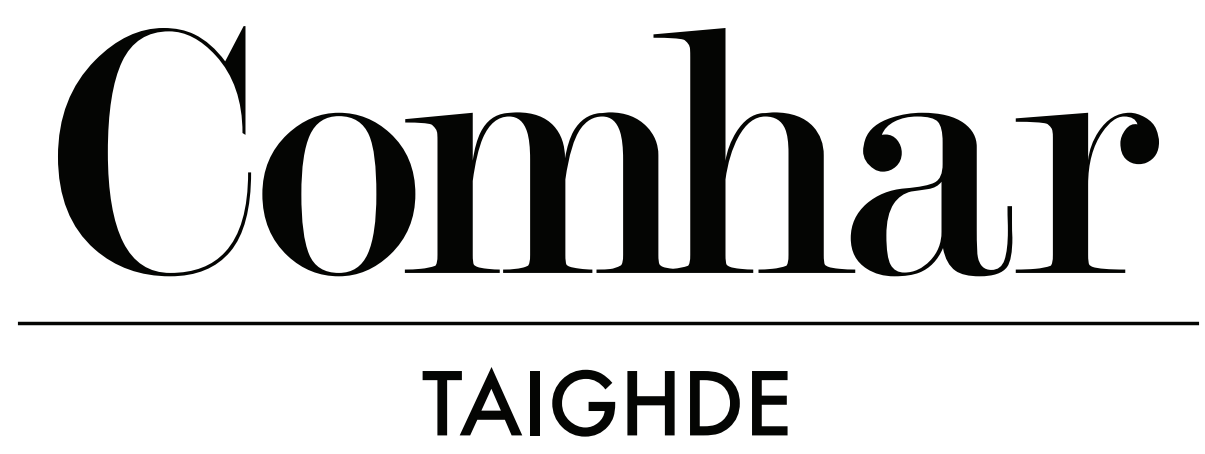

EAGRÁN 6

Samhain 2020

\begin{abstract}
Alt Taighde
'A bhean úd thíos ar bhruach an tsrutháin': an teanga fhíortha agus próiseas an deasghnátha i seoithín traidisiúnta ó Ghaeltacht Mhúscraí
\end{abstract}

Dáta foilsithe:

30 Samhain 2020

Cóipcheart:

(C) Tríona Ní Shíocháin, 2020 (CC BY-NC)

\section{Seoladh gréasáin:}

https://comhartaighde.ie/eagrain/6/nishiochain/
Údar:

Tríona Ní Shíocháin

\section{Comhfhreagras:}

triona.nishiochain@mu.ie

Seoladh seasmhach (DOI):

https://doi.org/10.18669/ct.2020.05

Is le cabhair deontais i gcomhair tograí Gaeilge a d'íoc an tÚdarás um Ard-Oideachas trí Choláiste na hOllscoile, Corcaigh le tacaíocht Choiste Léann na Gaeilge, Litríocht na Gaeilge agus na gCultúr Ceilteach, Acadamh Ríoga na hÉireann, a fhoilsítear an ríomhiris seo. Aithníonn COMHAR an tacaíocht seo.

\title{
comhartaighde.ie
}

ISSN: 2009-8626 


\section{'A bhean úd thíos ar bhruach an tsrutháin': an teanga fhíortha agus próiseas an deasghnátha i seoithín traidisiúnta ó Ghaeltacht Mhúscraí}

\section{Tríona Ní Shíocháin}

\section{Achoimre}

Déanfar plé sa pháipéar seo ar choincheap Bhean an Leasa mar atá le fáil sa seoithín traidisiúnta 'A bhean úd thíos ar bhruach an tsrutháin' ó Ghaeltacht Mhúscraí. Ba ghnáthchuid de chleachtais thraidisiúnta amhránaíochta é an seoithín a bhí ceangailte go dlúth le saol an teaghlaigh agus le cúram leanaí go háirithe. Bhí rian an deasghnátha agus na draíochta go láidir air (Ó Madagáin 1989, 1992) agus ba chleachtas cruthaitheach é dá réir sin a tuigtí mar chosaint ar an dainséar, agus a bhí nasctha go dlúth le tuiscintí an phobail ar an osnádúr. Ag tarraingt ar shaothar Mháire Ní Annracháin ar an teanga fhíortha i dtraidisiún na Gaeilge, agus ar shaothar Angela Bourke ar na scéalta sí, cíorfar an nasc idir an meafar, an saol eile, agus feidhm dheasghnáthaíoch na hamhránaíochta i gcás an tseoithín seo. Díreofar go speisialta ar an aistear tairseachúil a chruthaíodh an seoithín traidisiúnta don tsuibiacht bhaineann, ar an ionchollú trí mheán na hamhránaíochta ar bhean shamhailteach an leasa, agus ar an bhfeidhm ar leith a bhí le domhan fíorúil an amhráin mar le 'communitas' nach beag a chruthú idir mhná. Sholáthraigh dioscúrsaí trí mheán na hamhránaíochta ar an bhfuadach sí smaointeoireacht shofaisticiúil choincheapúil a chuir ar chumas na mban dul i ngleic le gnéithe fíordhúshlánacha dá saol. Ní hamháin go raibh tábhacht nach beag ag baint leis an seoithín traidisiúnta mar le teachtaireachtaí rúnda agus dioscúrsaí faoi cheilt a nochtadh agus a phlé i measc na mban, dá réir, ach d’fhág feidhm dheasghnáthaíoch an tseoithín teacht aniar ar leith sna mná in am an ghátair. 
Agus í ag scríobh ar cheist na teangan fíortha i litríocht na Gaeilge in 1998, dhein Máire Ní Annracháin léamh íogair ar choincheap an tsaoil eile i gcultúr agus i litríocht na Gaeilge. Seo mar a dúirt sí: 'go bhfeidhmíonn an saol eile go minic i litríocht na Gaeilge ar nós mar a d'fheidhmeodh meafar' (1998: 59). Sa pháipéar seo, ba mhaith liom forbairt a dhéanamh ar choincheap Ní Annracháin ar mheafaracht an tsaoil eile trí anailís a dhéanamh ar phearsa Bhean an Leasa i seoithín traidisiúnta ó cheantar Mhúscraí dar teideal 'A bhean úd thíos ar bhruach an tsrutháin'. D'áitigh Ní Annracháin gur mó cosúlacht idir an dá shaghas machnaimh seo, is é sin le rá, an meafar agus an saol eile, cé go bhfeidhmíonn ceann díobh ar leibhéal an chreidimh, agus an ceann eile i dtéarmaí ealaíne amháin, geall leis:

Smaoinigh go speisialta ar chumas an mheafair léim shamhlaíochta a ionchollú, agus rudaí a cheangal nó a chomhshamhlú atá difriúil ó chéile ach cosúil le chéile ag an am gcéanna; nó cosúlacht aduain nach n-aithnítear go coitianta a shamhlú; nó mar a bheadh domhan úrnua á chruthú. Feidhmíonn an saol eile go beacht agus go torthúil chun na rudaí seo a dhéanamh, ach déantar iad laistigh de rialacha a thuig an pobal, óir thuigeadar nósmhaireacht an tsaoil eile (1998: 59).

Áitím go bhfuil comhtháthú idir an meafar agus an saol eile le fáil sa tseoithín traidisiúnta seo ina n-insítear dúinn faoi eispéireas na mná a fuadaíodh agus atá anois i ndaorbhroid sa lios, í deighilte amach óna muintir agus ón domhan saolta araon. Murab ionann agus na scéalta a bhí i measc an phobail ar bhean an leasa, áfach, ina gcloistear de ghnáth faoin mbean a fuadaíodh sa tríu pearsa amháin, sa tseoithín seo cloistear faoi thaithí na mná seo trína súile féin (Bourke 1992: 86; Bourke 1995: 573). Ní hamháin go bhfuil an creideamh sa tsaol eile mar dhlúthchuid den amhrán, áfach, ach áitím go nglacann an t-amhránaí ionad na mná sa lios go sealadach agus go fíorúil le linn a ráite. Fágann san go mbeadh ionchollú á dhéanamh trí ghníomh na hamhránaíochta ar phearsa shamhailteach, cé gur mó ná pearsa fhicseanach na ngnáth-ghnásanna liteartha í, gan dabht, sa chás seo.
An bhean saolta a déarfadh an t-amhrán seo mar shuantraí dá páiste, bheadh sí ag cur síos ar chruachás mná eile, agus an chaoi a raibh sí ag iarraidh teacht as. Ach d'fhéadfadh sí bheith ag cur síos ar a cás féin ag an am céanna - agus an cleas ceannann céanna á imirt aici (Bourke 1992: 86).

Léiríonn saothar June Boyce-Tillman dúinn gur minic a shamhlaíonn an duine é féin i dtéarmaí samhailteacha trí shúile dhuine eile mar chuid d'eispéireas tairseachúil an cheoil (2009: 186). D'fhéadfadh an t-amhránaí sa chás seo ionannú láidir a dhéanamh le Bean an Leasa atá ag insint a scéil dúinn san amhrán, agus cás Bhean an Leasa a thuiscint i dtéarmaí a saoil féin. Go deimhin, baineann tábhacht le heispéireas an cheoil ó thaobh na suibiachtúlachta de go speisialta: 'Here the subjectivity of all those involved in the musical event intersect' (Boyce-Tillman 2009: 186). Áitím go dtarlaíonn trasnú idir suibiachtúlachtaí na mban saolta agus samhailteach ar an gcuma chéanna seo i gcás an tseoithín.

Níorbh ionann agus 'ficsean' na scéalta sí, áfach, ar an ábhar go raibh blas na fírinne go láidir orthu mar chuid den chreideamh coiteann, mar a mhíníonn Angela Bourke dúinn: 'Ní "fairytales" atá i gceist anseo, ach scéalta seanchais faoi theagmháil a bheith ag daoine daonna leis an slua sí, scéalta a chuirtear i láthair mar thuairiscí fírinneacha' (1992: 78). Tugann tráchtaireacht Eoghain Uí Chomhraí le fios dúinn gurb amhlaidh do phearsa bhean an leasa san amhrán seo leis (Petrie 2002 [1855]: 109). Sa tslí seo, chítear go bhfuil an domhan saolta, an domhan neamhshaolta, agus meafaracht na hamhránaíochta ag sileadh isteach ina chéile i slí an-éifeachtach san amhrán.

Creidim, dá réir sin, go léiríonn an seoithín seo go bhfuil dlúthnasc idir feidhm an mheafair agus brí an tsaoil eile sa tsochaí thraidisiúnta, mar a d'áitigh Ní Annracháin. Tá gréasán coimpléacsach coincheapúil le fáil san amhrán go mba dhlúthchuid de thraidisiún smaointeoireachta na mban agus de thraidisiún rúnda cumarsáide ina measc é (Bourke 1992: 80). Ní hamháin go bhfaightear tuiscintí coimpléacsacha ón gcreideamh coiteann ag meascadh le híomhánna samhailteacha an amhráin, ach dearbhaím, i bhfianaise taighde atá foilsithe cheana féin agam, go bhfeidhmíonn gníomh na hamhránaíochta féin mar a bheadh 
deasghnáth ann (Ní Shíocháin 2012: 49-78; Ní Shíocháin 2018: 23-55), deasghnáth a chuireann ar chumas na máthar teacht slán trí aistear priaclach nó trí thréimhse chruatain ina saol féin. Creidim go raibh ionad ar leith ag dioscúrsaí na mban samhailteach san amhrán i gcruthú an fhrithstruchtúir ('anti-structure' Turner 1969; Abrahams 2017: x) i saol na mban daonna, rud a chumasaigh plé ar ghnéithe fíordhúshlánacha de shaol na mban (Nic Cárthaigh 2002: 66-68; Ó Laoire 2016: 6-7). Áitím dá réir sin go bhfaightear léiriú suntasach sa tseoithín traidisiúnta seo ar thraidisiún imeallach smaointeoireachta lenar bhain aistear sacrach don tsuibiacht bhaineann sa tsochaí thraidisiúnta. Is é eispéireas sin na suibiachta laistigh de dhomhan fíorúil an amhráin is suim liom anseo: aistear tairseachúil, deasghnáthach na hamhránaíochta a bhí mar theacht aniar do mhná agus iad ag dul i ngleic le deacrachtaí ina saol.

\section{Cás na mná in 'A bhean úd thíos ar bhruach an tsrutháin'}

Is ar leagan ó Mhúscraí a dhíreod anseo, cé gur bailíodh leagan an-chosúil leis ó cheantar Luimnigh i lár an naoú haois déag (Petrie 2002 [1855]: 106-109), agus gur bailíodh seoithín atá gaolmhar leis ó thaobh ábhair de ó Mháire Bean Uí Cheallaigh ón mBaile Mór i nDún na nGall a foilsíodh in Siscéalta ó Thir Chonaill (Ó hEochaidh et al. 1977: 66-68). Mar sin, is dócha gur téama agus amhrán ab ea é seo a mbíodh leaganacha de le fáil coitianta ar fuaid na hÉireann tráth. Is iad na focail mar a thugann Eilís Ní Shúilleabháin ó Chúil Aodha uaithi iad ar a dlúthdhiosca Cois Abhann na Séad (1997; Ó Conghaile et al. 2012: 51-52) a phléifear thíos, lena mbaineann an fonn céanna ceoil a bheag nó a mhór a bailíodh ó Bhess Cronin in 1947 (Ó Cróinín 2000: 197-200). Pléifear chomh maith an míniú a thugann Bess Cronin féin ar an amhrán agus í á phlé le hAlan Lomax in 1951.

Is píosa filíochta an seoithín traidisiúnta seo atá dea-shnoite dea-chumtha, agus sampla is ea é de chleachtas cruthaitheach sofaisticiúil a forbraíodh agus a mhair idir mhná i gcomhthéacs an tí agus an teaghlaigh. Línte gearra filíochta a fhaightear san amhrán a thugann cur síos dúinn ar scéal corraitheach agus truamhéileach na mná. Críochnaítear gach líne den amhrán le friotal foirmleach an tseoithín thraidisiúnta - 'seoithín seó, uil leó leó. Is ar a gcuma seo a fhaighimid amach de réir a chéile cad é atá i ndán don bhean atá ag labhairt, agus cruthaíonn sé seo teannas nach beag san amhrán trí chéile. Ní go dtí deireadh an amhráin a thuigimid an phráinn agus an dainséar a bhaineann le cás na mná seo: tá an t-am ag breith uirthi agus tá baol láidir ann gur go brách na beithe a bheidh sí ina príosúnach sa lios.

Tá braistint ghiorranálach fiú amháin le sonrú ar an tslí ina gcuirtear scéal an amhráin i láthair, mar go soláthraíonn an 'seoithín seó, uil leó leó' sosanna nádúrtha i struchtúr na hinsinte, rud a chuireann leis an impí chráite atá á déanamh ag an mbean ar an éisteoir:

A bhean úd thíos ar bhruach a' tsrutháin, seoithín seó, uil leó leó,

Is an dtuigeann tusa fáth mo ghearáin, seoithín seó, uil leó leó,

Mar is bliain's an lá so a tógag mé óm leannán, seoithín seó, uil leó leó,

Is do rugag mé 'steach go lios a' chnocáin, seoithín seó, uil leó leó,

Seoithín, seoithín, seoithín, seoithín, seoithín seó, uil leó leó,

Seoithín, seoithín, seoithín, seoithín, seoithín seó, uil leó leó.

(Ní Shúilleabháin 1997: Traic 12; is liomsa an téacs tras-scríbhte).

Labhrann an bhean le bean eile atá thíos ar bhruach an tsrutháin, agus is i gcogar agus faoi rún a insíonn sí a scéal uafásach di. Deintear cur síos ar an saol sa lios, ina bhfaightear idir áilleacht agus dhiamhaireacht, ina dtugtar nod dúinn go bhfuil rud éigin as alt nó bagairt chiúin rúndiamhair éigin laistiar den fhlúirse agus den fhlaithiúlacht a chuirtear os ár gcomhair:

Ag seo anso mo theach mór maiseach ...

Is mó leann buí agus leann sean ann ...

Is mó mil bhuí agus céir bheach ann ...

Is is mó seanduine ar a nasc ann ...

(Ní Shúilleabháin 1997: Traic 12; is liomsa an téacs tras-scríbhte).

Is seandaoine iad seo a fuadaíodh agus iad óg, b’fhéidir, iad san nár éirigh leo éalú as an lios ó shin (Petrie 
2002 [1855]: 110). Tá feo laistiar den fhlúirse agus den mhaise, agus tá an baol ann gur mar seo a bheidh anois don bhean seo go deo. De réir mar a ghluaiseann an t-amhrán ar aghaidh, cloisimid go bhféadfaí í a shaoradh, ach deasghnáth ar leith a chur i gcrích:

Is abair lem chéile tíocht amáireach ...

Is an choinneal chiarach i gcúl a dhearnainn' ...

Is an scian choise duí a thúirt'na láimh leis ...

Is an capall tosaig a bhuala sa bhearnain ...

(Ní Shúilleabháin 1997: Traic 12; is liomsa an téacs tras-scríbhte).

Glacaim leis go n-aithneodh an pobal éisteachta na céimeanna seo go léir, ag brath ar an traidisiún áitiúil a bhí chun cinn sa cheantar, ach is dócha gurb í an tagairt do scian na coise duibhe is mó a thuigfí, mar go mba chosaint thraidisiúnta é an t-iarann agus scian na coise duibhe go háirithe ar na sióga (Petrie 2002 [1855]: 110). Sa véarsa deireanach de leagan Eilís Ní Shúilleabháin, tuigtear dúinn go mbeidh ar fhear na mná teacht agus luibh speisialta a bhaint ag doras an leasa. Mura n-éiríonn leis, is sa lios in aimsir ag an slua sí a bheidh an bhean seo feasta: 'Nó mura dtí sé ag a' dtráth san ... Go mbeadsa im banrín ar na mná so .... Cruthaíonn doircheacht an dioscúrsa seo agus an sceimhle croí a bhaineann le cás na mná codarsnacht an-suimiúil agus é taobh leis an ngluaiseacht chun suaimhnis a bhaineann le fonn ceoil agus feidhm an tseoithín. Mar a thuigimid ó Bhess Cronin, seoithín ab ea é seo a bhíodh á rá agus páistí á gcur a chodladh i nGaeltacht Mhúscraí, agus tá cosaint shéimh agus cion le brath go láidir ó na línte foirmleacha 'seoithín seó ...' go háirithe (Nic an Airchinnigh agus Ó Laoire 2014: 165-166). Is ar an gcuma seo atá brí faoi cheilt le brath láithreach ar na dioscúrsaí san amhrán, brí atá ró-dhorcha le go dtuigfeadh líon óg an tí í go hoscailte. In agallamh a chuir Alan Lomax ar Bhess Cronin in 1951 mar gheall ar an amhrán seo, fiafraíonn sé de Bhess cad chuige an t-amhrán:

Alan Lomax: That is one of the most beautiful songs I ever heard. What's it for?

Bess Cronin: Well, I'll give you the meaning of it ... it is, like, you'd sing it for a child if you were pacing the floor now say with a child to get him to go to sleep ... and you should sing something to the child of course ... (Bailiúchán Alan Lomax: T3283.0,

Traic 7).

Cuid lárnach de bhrí na bhfocal mar sin ná comhthéacs an teaghlaigh - bíonn dlúthbhaint ag an amhrán le máithreacha agus leanaí atá suaite nó cráite á gcur chun suain acu. Amhrán is ea é a bhaineann le 'pacing the floor' go sonrach i bhfocail Bess féin thuas, nó le bheith ag siúl agus ag siúl ar mhaithe le leanbh atá corraithe cráite a chiúnú. Samhlaím, mar sin, gurb amhlaidh a shilfeadh eispéireas na máthar isteach i mbrí na bhfocal, agus go silfeadh an cruachás ar leith a bhaineann le tuirse gan teorainn an chúraim seo isteach i meafar an tseoithín féin. Mar sin ní hamháin go dtagraíonn meafar Bhean an Leasa do ghréasán tuiscintí cosmeolaíocha atá nasctha go dlúth le heispéireas imeallach na mban, ach ritheann taithí na mban a deireadh an seoithín, agus iad i mbun cúraim leanaí, isteach i ndomhan meafarach an amhráin chomh maith. Samhlaím, mar sin, dála Angela Bourke, gur teachtaireachtaí rúnda ab ea iad seo idir mhná agus iad i mbun a gcúraimí laethúla, chun go ndéanfaí cumarsáid le máithreacha eile i ngan fhios don ghlúin óg, nó go deimhin i ngan fhios d'fhear an tí. Mar a d'inis Bess Cronin do Alan Lomax in 1951 faoin mbean san amhrán, 'She knew the woman would understand' (Bailiúchán Alan Lomax, T3283.0, Traic 7), agus b’fhéidir gurbh amhlaidh do na gnáthmhná a deireadh na seoithíní seo chun a chéile i gcomhthéacs tí chomh maith céanna - máithreacha, máithreacha críonna, iníonacha lánfhásta, aintíní agus araile. Dealraíonn sé go bhféadfadh comhtháthú thar a bheith spéisiúil tarlúint dá réir sin idir na mná samhailteacha i scéal an amhráin agus na mná a bhíodh ag rá na n-amhrán. Is sampla é seo den 'c[h]osúlacht aduain nach n-aithnítear go coitianta a shamhlú' a bhaineann le feidhm an mheafair agus feidhm an tsaoil eile araon (Ní Annracháin 1998: 59).

Mar a áitíonn Ní Annracháin, bíonn brí na teangan siombalaí ag brath go minic ar réamhthuiscintí an phobail (1998: 52), mar a chítear i ndomhan coincheapúil an amhráin áirithe seo. Baineann dioscúrsaí na scéalta sí go speisialta le daoine imeallacha, nó tréimhsí nó staideanna tairseachúla i saol an duine, mar atá léirithe ag Bourke: 'Fairies belong to the margins, and so can serve as reference points for all 
that is marginal in human life' (1999: 28). Dealraíonn sé, dá réir sin, go mb'fhéidir gur domhan siombalach is ea an creideamh sna sióga gur dioscúrsa meafarach ar eispéiris thairseachúla an duine féin é chomh maith - 'narratives of passage analogous to rites of passage', mar a dúirt Bourke (1995: 570). Sna síscéalta féin, is minic gur mná a bhíonn ag teacht trí thréimhse thairseachúil ina saol féin a luaitear in eachtraí a bhaineann leis an slua sí - mná ag breith leanaí, mná cabhartha, nó mná nuaphósta a bhfuil stádas nua anois bronnta orthu ina saol pearsanta féin. Uaireanta eile faightear cur síos sna síscéalta ar mhná a bhfuil breoiteacht mhistéireach nach féidir a leigheas tagtha orthu:

Ní amháin go gcailltí mná agus páistí i dtinneas clainne, agus gur ar éigean a tháinig cuid eile acu slán: bhí taobh síceolaíoch leis an scéal freisin. An bhean a chaillfí i dtinneas clainne, d'fhéadfaí a bás a mhíniú, mar a mhínítí bás anabaí i gcoitinne mar fhuadach sí, ach an galar a dtugtar 'postnatal depression'i mBéarla air, d'fhéadfaí cur síos go héifeachtach air sin freisin i dtéarmaí na síceolaíochta seo. Níorbh í an bhean féin í - an créatúr cantalach nach raibh ag déanamh aon mhaith ó rugadh an páiste, nó ó cailleadh é - ach iarlais a bhí curtha ina háit ag an slua sí (Bourke 1992: 82).

Coincheap is ea an fuadach sí, dá réir sin, a insíonn scéal dúinn faoi eispéireas an tsaoil dhaonna laistigh den insint ar an slua sí agus ar an saol eile. Córas creidimh a fheidhmíonn mar a bheadh meafar ann, mar a d'áitigh Ní Annracháin, 'arbh í an chosúlacht a bunchloch' (1998: 37). B'fhéidir go n-insíonn coincheap an fhuadaigh agus coincheap na hiarlaise, cuir i gcás, gur rud leochaileach is ea sláinte an duine, agus go maireann an tsuibiacht i ndomhan priaclach nach bhfuil smacht iomlán ag an duine féin air. Nó b’fhéidir gur léiriú casta é ar imeallú na mban laistigh den tsochaí nó laistigh de réimeas na bhfear ag leibhéal pobail nó teaghlaigh, nó ar réimse leathan aicídí ón eitinn go dtí an ísle brí (Bourke 1995: 571).

Gné eile de na síscéalta ná na gníomhartha foréigneacha a ruaigeann an iarlais agus a 'shábhálann' an bhean shaolta ón domhan osnádúrtha. D’fhéadfaí é seo a thuiscint mar smachtú nó stiogmatú sóisialta a raghadh i bhfeidhm go mór ar chleachtais agus ar iompar daoine sa tsochaí thraidisiúnta:
Even if changeling beliefs did not usually lead to attacks on adult women, they would have encountered its verbal sanctions and imaginative possibilities at every point of stress in their lives. The anxieties of social life might find expression in changeling accusations, but might also give rise to claims of changeling status. Some fairy legends may certainly be read as expressions of the loneliness and alienation of young women married by the decisions of older men (Bourke 1995: 571).

Meafar creidmheach is ea Bean an Leasa a thagraíonn d'eispéireas coimpléacsach na mban laistigh d'ord na bhfear sa tsochaí thraidisiúnta. Anuas air seo, áfach, samhail is ea Bean an Leasa a léiríonn blas na teagmhála dainséaraí a bheith ar an saoldearcadh traidisiúnta. Meabhraíonn sé seo sanasaíocht an fhocail 'eispéireas' dom mar atá léirithe ag Turner (1985: 85), ina gciallaíonn 'per' an fhocail 'experience', 'taisteal tríd': gur rud is ea an t-eispéireas atá éiginnte agus mistéireach, rud a gcaithfear gabháilt tríd, agus a mbaineann ciall an 'rite de passage' go dlúth leis (Thomassen 2014: 86). Dearbhaím gur slí ar leith ab ea an seoithín chun deasghnáthú a dhéanamh ar na dúshláin eiseacha sin i saol na mban, a lig dóibh gabháilt tríd an gcruatan trí aistear amhránaíochta a thug isteach i ndomhan fíorúil smaointe iad ar feadh scaithimh, agus a thug slán abhaile thar n-ais arís ar ball iad. Ba léir go raibh teacht aniar sa tseoithín.

\section{Ag gabháilt tríd agus an teacht aniar: aistear tairseachúil an tseoithín thraidisiúnta}

Ar an ábhar go mbaineann cosaint dhraíochta le friotal foirmleach an 'genre' seo go traidisiúnta, dar le Breandán Ó Madagáin (1989: 33-35; 1992: 133), d'fhéadfaí a rá gur gnás draíochta ann féin é an seoithín a thagann le cur síos van Gennep ar dheasghnátha draíochta go hidirnáisiúnta (1960: 8-14). Mar atá pléite agam in áiteanna eile, creidim go gcruthaíonn cleachtas na hamhránaíochta spás tairseachúil i saol an duine a bhfuil cosúlachtaí láidre idir é agus próiseas an deasghnátha (van Gennep 1960: 10-11, 65-115; Turner 1969: 94-130; Ní Shíocháin 2018: 31-34, 41-51; Ó Laoire 2002: 208-210). Áitím gur cleachtas is ea an seoithín traidisiúnta, dá réir 
sin, a thógann an t-amhránaí amach as an ngnáthshaol ar feadh tamaillín, agus ina bhfuil deighilt, idirthréimhse, agus comhshnaidhmeadh an 'rite de passage', mar a thrácht van Gennep orthu, le sonrú (1960: 10-11). Cruthaíonn gníomh na hamhránaíochta frithstructhúr ina leánn gnáthstruchtúir agus gnáthrialacha an tsaoil ar feadh tréimhse, agus mar a d'áitigh Máirín Nic Eoin, is é an ceol féin a chruthaíonn an comhthéacs ar leith 'a shaorann an teanga ó laincisí na gnáthchumarsáide laethúla' (2000: 233). Soláthraíonn struchtúr solúbtha aeistéitiúil na rithime agus an cheoil frithstruchtúr fuaime don éisteoir agus don amhránaí, a chruthaíonn eispéireas ama atá bunoscionn leis an ngnáth-am (Ní Shíocháin 2018: 26-29). Anuas air seo, is léir go mbaineann tairseachúlacht le hoidhreacht leis an bhfilíocht féin sa traidisiún Gaelach ó thréimhse na luathlitríochta anuas go dtí traidisiún béil na Nua-Ghaeilge (Nagy 1985: 21-26; Ó hÓgáin 1982: 5-6, 93-103; Ó Riain 1971-1972; Partridge 1980). Fágann san gur siombailí cumhachtacha traidisiúnta iad an mheadaracht, an rithim, agus an ceol in aonacht a chruthaíonn an tairseachúlacht. Ní hionann dá réir sin domhan an tseoithín agus domhan an ghnáthshaoil. Ligeann sé seo do dhioscúrsaí dúshlánacha teacht chun cinn, agus glacann an pobal leis an dánaíocht nó leis an imreas nó leis an gcur trí chéile a chruthaítear i bhfrithstruchtúr na hamhránaíochta nach nglacfaí chomh saoráideach leo i réimsí eile den saol (Ní Shíocháin 2018: 41-51). Baineann tréithe suntasacha leis an tairseachúlacht i slite eile chomh maith sa mhéid is go mbronntar cumhachtaí ar íochtaráin go sealadach mar chuid d'idirthréimhse phróiseas an deasghnátha go coitianta (Turner 1969: 100-103, 109-111). Mar shampla, is minic a cheadaítear don dream atá thíos leis tabhairt faoi lucht cumhachta i bhfoirm amhránaíochta, cé nach gceadófaí a leithéid sa ghnáthchaint, mar a chítear i dtraidisiúin bhéil de chuid na mban ar fuaid an domhain (Ní Shíocháin 2018: 41-42). Anuas air seo, mar gheall ar thréithe na tairseachúlachta a bheith ag baint léi mar chleachtas, slí is ea an amhránaíocht chun deasghnáthú a dhéanamh ar eispéireas an tsaoil, rud a chabhraíonn le daoine dul i ngleic le deacrachtaí pearsanta agus uile agus teacht chucu féin arís ina dhiaidh (Boyce-Tillman 2009; Ní Shíocháin 2018: 127).
Laistigh den fhrithstruchtúr amhránaíochta seo, mar sin, is ea a bhí cead aighnis ag na mná, nó cead cainte ar ghnéithe dorcha tromchúiseacha dá dtaithí saoil féin. Trí ghníomh an taibhléirithe i ngnáthsaol an teaghlaigh is ea a d'aimsigh na mná seo straitéisí le teacht slán trí eispéiris imeallacha thairseachúla ina saol féin. Ina chroílár sin bhí an t-ionchollú trí ghníomh na hamhránaíochta, agus i réimse na samhlaíochta agus an chreidimh araon, a dheineadh an bhean shaolta ar bhean an leasa san amhrán. Fágann san gur meafar sacrach nó naofa nó deasghnáthach is ea samhail Bhean an Leasa, meafar nach maireann sa bhfilíocht amháin ach i gcosmeolaíocht dhúchais na ngnáthdhaoine chomh maith. Meafar is ea Bean an Leasa a shíneann amach as an gcleachtas cruthaitheach agus isteach i gcúrsaí an ghnáthshaoil i slí an-doimhin; mar a dúirt Angela Bourke: 'Is é atá in an-chuid den sísheanchas, dar leis an tuiscint seo, bealach ag mná le rudaí a rá go hindíreach, nó go meafarach, nárbh fhéidir a rá go hoscailte: rudaí nach ligfí dóibh a rá ar aon bhealach eile' (1992: 85-86). Tá cumhacht ar leith ag baint leis na teachtaireachtaí ceilte seo atá le fáil in insint bhéil na mban a chruthaíonn nasc láidir idir mhná mar ghrúpa dá réir (Ó Laoire 2016: 13). Mar an gcéanna atá dioscúrsaí na hamhránaíochta, dar ndóigh: deis ab ea an seoithín chun fírinní crua a nochtadh faoi cheilt.

Anuas ar chumas na gcleachtas tairseachúil seo coincheapa a mhúscailt a d'fháisc brí as dúshláin an tsaoil do mhná, d'fhéadfaí a rá go raibh ról ar leith ag tairseachúlacht na hamhránaíochta maidir le cruthú gníomhúil na suibiachta féin. Ba í an ghníomhúlacht seo chomh maith a chruthaigh cad ba bhrí agus ba bhunús leis an mbean agus leis an mbaineann sa tsochaí thraidisiúnta (Butler 1988: 519-520). Toisc feidhm an smachta a bheith chomh lárnach sna síscéalta, díol suime is ea é gurb í an bhean í féin a labhrann linn sa tseoithín seo (Bourke 1995: 572-573). Cleachtas cultúrtha is ea an seoithín a bhaineann go sonrach le saol na mban, agus áitím go gcruthaíonn eispéireas tairseachúil na hamhránaíochta, ina samhlaíonn an t-amhránaí í féin mar bhean an leasa go sealadach, 'communitas' láidir idir mhná sa tsochaí thraidisiúnta, rud a bheadh ina fhál cosanta 'i saol a bhí faoi réimeas na bhfear' (Ó Laoire 2016: 13).

Comhnascadh láidir daonna nó comhthuiscint dhoimhin a eascraíonn as 'communitas' na tairseach- 
úlachta dar le Victor Turner (1969). Mar a d'áitigh sé agus é ag trácht ar ghrúpaí daoine a loirgíonn cothú daonna an 'communitas' ina saol féin: 'What they seek is a transformative experience that goes to the root of each person's being and finds in that root something profoundly communal and shared' (Turner 1969: 138). I seoithíní ar nós 'A bhean úd thíos ar bhruach an tsrutháin', glacann an bhean a deir an t-amhrán ionad bhean an leasa go samhailteach agus sileann a taithí féin ar an imeallú agus ar dhúshláin an mháithreachais isteach i mbrí an amhráin. Deineann an t-amhránaí ionannú leis an mbean san amhrán, agus deineann sí nascadh le pobal ban a thuigeann go dlúth agus go smior, más faoi rún féin é, an bhrí atá le dioscúrsa an amhráin. Nó, i dtéarmaí Victor Turner, aimsíonn an t-amhránaí comhthuiscint dhoimhin sa tseoithín a bhaineann go dlúth le heispéireas coiteann na mban laistigh d'ord na bhfear; mar a dúirt Bess Cronin: 'She knew the woman would understand' (Bailiúchán Alan Lomax, T3283.0, Traic 7).

Seo mar a dúirt Turner faoi fheiniméan an 'communitas' le linn na tairseachúlachta:

Communitas breaks in through the interstices of structure in liminality; at the edges of structure, in marginality; and from beneath structure in inferiority. It is almost everywhere held to be sacred or 'holy', possibly because it transgresses or dissolves the norms that govern structured and institutionalized relationships and is accompanied by experiences of unprecedented potency (1969: 128).

Áitím gur eascair 'communitas' ar leith idir mhná as cleachtas traidisiúnta an tseoithín - ba é an seoithín an 'scoilt' sa ghnáthstruchtúr laethúil a lig do 'communitas' na mban teacht chun cinn. 'Communitas' ón íochtar nó 'from beneath structure in inferiority'ab ea é seo, mar a chuir Turner thuas é. Leáigh an seoithín na noirm a rialaigh struchtúr sóisialta an teaghlaigh agus na sochaí traidisiúnta, ar feadh scaithimh ar aon nós.

Dlúthchuid den eispéireas tairseachúil seo ab ea an teanga fhíortha féin a chruthaigh domhan coincheapúil Bhean an Leasa, rud a lig do mhná cíoradh a dhéanamh ar an eispéireas baineann trí mheán an mheafair agus na cosmeolaíochta araon. D'fhág gníomh na hamhránaíochta go bhféadfaí brí litriúil na bhfocal a chur ar ceal, mar a dhéanfadh meafar (Ní Annracháin 1998: 35), agus domhan eile a shiúl ar feadh tamaill a d'fháisceadh brí as eispéireas na beatha.Meán ab ea an amhránaíocht a d'fhorbair córas smaointeachais a chruthaigh leibhéil éagsúla mheafarachta a dhein taithí na mban, domhan fíorúil baineann, agus dioscúrsaí rúnda na mban a fhí ar a chéile go héifeachtach. Bhí tábhacht ar leithligh le gné an cheoil, go mba dhlúthchuid d'eispéireas smaointeoireachta na hamhránaíochta í, a lig don amhránaí (nó don éisteoir) éalú ó ghnáthrithimí an tsaoil ar feadh scaithimh agus dul i ngleic leis an tocht agus leis an mbrí i slí an-doimhin. Tá tábhacht mhothúchánach na n-amhrán i gcomhthéacs pobail léirithe ag Ó Laoire (2002: 208-212), a insíonn dúinn gur tuigeadh do dhaoine fiú amháin 'go raibh toise ar leith san amhránaíocht nach raibh breith ar bith ag an scéalaíocht uirthi' (2002: 206-207). Díol suntais is ea an tslí ina dtéann an ceol, an rithimiúlacht agus an mheadaracht i bhfeidhm ar eispéireas an ama féin, rud a chruthaíonn spás ar leith 'ina dtig an aimsir chaite agus an aimsir fháistineach le chéile in aon aimsir láithreach shínte amháin, a dhéanas iarracht an gnáth-am a chur droim ar ais ar feadh tamaill' (Ó Laoire 2002: 207). Áitím gur chumasaigh eispéireas an cheoil caidreamh coimpléacsach mothúchánach (Jaimovich et al. 2013: 20) le domhan meafarach an amhráin: is amhlaidh a bhí an ceol agus an meafar ag cothú a chéile, agus go mba dhlúthchuid de bhrí an amhráin an nasc sin eatarthu.

Samhlaítear dom, dá réir sin, go raibh ionad lárnach ag an teanga fhíortha in 'A bhean úd thíos ar bhruach an tsrutháin' i gcruthú na hidirthréimhse tábhachtaí seo i saol na mban. Aistear tairseachúil go dtí domhan fiorúil de chuid na mban ab ea an seoithín a ligfeadh dóibh gabháilt tríd an bhfulaingt, agus teacht aniar arís agus sólás fachta acu ó 'communitas' na mban samhailteach agus daonna araon. Mar a déarfadh Boyce-Tillman (2009: 185-186, 191-197), is amhlaidh a chruthaigh an tairseachúlacht seo faoiseamh agus athbheochan tuisceana don amhránaí agus dhaingneofaí an teacht aniar a bhí inti dá réir. Frithbheartaíocht chiúin de chuid na mban ab ea domhan fiorúil rúnda an tseoithín. 


\section{Conclúid: Meafaracha agus Deirfiúracha}

Alt ceannródaíoch i Léann Inscne agus Feimineach na Gaeilge ab ea 'Ait liom bean a bheith ina file' a d'fhoilsigh Máire Ní Annracháin i Léachtai Cholm Cille in 1981 (Nic Dhiarmada 1992: 164; Nic Eoin 1998: xiv). Is mó ceist spreagúil a chuireann an t-údar ar lucht léinn san alt sin, ach is ar cheann díobh go háirithe a bhaineann go sonrach le ceist an tseoithín ar mhaith liom filleadh uirthi anseo:

An amhlaidh nár mhian leis na mná, nó nár éirigh leo, nó nár ceadaíodh dóibh dul i mbun an sórt cumadóireachta a nglactaí leis mar ardlitríocht de réir caighdeán an ghrúpa ceannais agus ina dhiaidh sin, an bhfuil traidisiún eile litríochta murar liteartha féin, a bhfuil cáilíochtaí agus slatanna tomhais dá chuid féin aige ar ar fhágadar a rian go soiléir? (Ní Annracháin 1981: 147; an bhéim sa bhuntéacs)

Creidim go dtugann traidisiún béil na Gaeilge léargas ar leith dúinn ar shaothair chruthaitheacha na mban Gaelach, agus go léiríonn 'A bhean úd thíos ar bhruach an tsrutháin', agus seoithíní eile mar é, stair smaointeoireachta a chleacht na mná a bhain go speisialta lena dtaithí saoil féin, nó 'traidisiún eile liteartha murar liteartha féin é’ i bhfocail Ní Annracháin thuas. Traidisiún smaointeoireachta ab ea é seo lenar bhain idir cheol agus choincheapa fileata, chomh maith le comhthéacs saibhir idirphearsanta an taibhithe. (Díol suime is ea argóintí Nic an Airchinnigh agus Uí Laoire (2014: 167-173) gur bhain scála módúil ar leith le foinn cheoil áirithe a bhí nasctha go dlúth le hobair agus le ról na mban sa tsochaí thraidisiúnta, rud a thabharfadh le fios gurbh ann do chleachtais cheoil ar leith a bhain leis na mná go speisialta agus a bhí coitianta ina measc). Mar atá léirithe ag Angela Bourke cheana féin, gréasán sofaisticiúil machnaimh ab ea na dioscúrsaí ar an slua sí, ar fhuadach ban agus leanaí, agus ar iarlaisí sa tsochaí thraidisiúnta (1992: 81-90; 1995: 568-573). Is léir gur traidisiún machnaimh é seo a bhí bunoscionn le luachanna na 'réasúnaíochta' a tháinig chun cinn ó ré na heagnaíochta i leith atá chomh lárnach sin i dtaibhléiriú na cumhachta sa tsochaí ó shin. 'Traidisiún ceilte', mar a dúirt Ní Annracháin (1981: 149), a chleacht na mná.
Traidisiún smaointeoireachta de shaghas é seo a bhí coimpléacsach agus a raibh an-saibhreas coincheapúil ag baint leis. Meafaracht agus cosmeolaíocht agus fírinne a fhitear ar a chéile i ndioscúrsaí an tseoithín, sa tslí is gurbh fhoinse athnuachana, foinse machnaimh, agus foinse 'communitas' agus dlúthpháirtíochta é a d'fhág teacht aniar sna mná in am an ghátair. Bhí brí agus cumhacht i dteangain fhíortha an tseoithín, gur chuid speisialta d'oidhreacht chruthaitheach agus intleachtúil na mban í. Dúirt Nietzsche tráth gurb é atá sa bhfírinne ná 'a mobile army of metaphors' (1954: 46), agus sa chás seo bhí meafar sacrach Bhean an Leasa mar arm ag mná ar an imeall, agus mar chosaint ar imreas cumhachta na sochaí patrarcaí.

Ceist íogair ábharach eile a chuir Ní Annracháin ar scoláirí na Gaeilge ná 'Cá bhfuil deirfiúracha Mháire Bhuî?’ (1981: 155). Áitím go n-aimseofar na deirfiúracha sin mar údair gan ainm i dtraidisiún na hamhránaíochta agus na filíochta, agus aimseofar iad i measc na mílte banamhránaithe stairiúla a chnuasaigh agus a chleacht amhráin ó bhéal ina ngnáthshaol laethúil. Guthanna na ndeirfuúracha sin atá le fáil sa tseoithín ó cheantar Mhúscraí a pléadh san alt seo. B'fhéidir gur amhránaíocht seochas litríocht ba ghnáthach leo, ach bhí an ealaín a chleachtadar chomh sofaisticiúil, más éagsúil féin í, le haon saothar liteartha. 


\section{SAOTHAIR A CEADAÍODH}

Abrahams, R.D. (2017) 'Foreword'. In: Turner, V., The Ritual Process: Structure and Anti-structure. Londain \& Nua-Eabhrac: Routledge: v-xii. Bailiúchán Alan Lomax, Lárionad Daonearraí Mheiriceá (American Folklife Center, Library of Congress), le caoinchead ón gComhlachas um Chothromas Cultúrtha (Association for Cultural Equity). Ar fáil ag https://archive.culturalequity. org/node/56333 [faighte 12 Aibreán 2021].

Bourke, A. (1992) 'Bean an Leasa: Ón bPiseogaíocht go dtí Filíocht Nuala Ní Dhomhnaill'. In: Ó hAnluain, E. (eag.) Leath na Spéire. Baile Átha Cliath: An Clóchomhar: 74-90.

Bourke, A. (1995) 'Reading a Woman's Death: Colonial Text and Oral Tradition in NineteenthCentury Ireland'. In: Feminist Studies 21 (3): 553-586.

Bourke, A. (1999) The Burning of Bridget Cleary. Auckland: Pimlico.

Boyce-Tillman, B. (2009) 'The Transformative Qualities of a Liminal Space Created by Musicking'. In: Philosophy of Music Education Review 17 (2): 184-202.

Butler, J. (1988) 'Performative Acts and Gender Constitution: An Essay in Phenomenology and Feminist Theory'. In: Theatre Journal 40 (4): 519-531.

Jaimovich, J. et al. (2013) 'Emotion in Motion: a Study of Music and Affective Response'. In: Aramaki, M., Barthet M., Kronland-Martinet, R. agus Ystad, S. (eag.) From Sounds to Music and Emotions. Heidelberg, New York, Dordrecht \& London: Springer: 19-43.

Nagy, J.F. (1985) The Wisdom of the Outlaw: the Boyhood Deeds of Finn in Gaelic Narrative Tradition. Berkeley: University of California Press. Ní Annracháin, M. (1981) 'Is Ait Liom Bean a Bheith ina File'. In: Ó Fiannachta, P. (eag.) $N a$ Mná sa Litríocht. Léachtaí Cholm Cille XII. Maigh Nuad: An Sagart: 145-182.

Ní Annracháin, M. (1998) 'An Teanga Fhíortha'.

In: Ní Annracháin, M. agus Nic Dhiarmada, B. (eag.) Téacs agus Combthéacs: Gnéithe de Chritic na Gaeilge. Corcaigh: Cló Ollscoile Chorcaí: 34-63.
Nic an Airchinnigh, M., agus Ó Laoire, L. (2014)

'Caointe agus Amhráin Chrúite: "Is le gach Bó a Lao agus le gach Caoineadh a Cheol"'. In: Aiste 4 (1): 155-176.

Nic Cárthaigh, G. (2002) 'Cúl le Cleamhnas'. In: Béascna 1: 59-72.

Nic Dhiarmada, B. (1992) “Ceist na Teanga” - Dioscúrsa na Gaeilge, an Fhilíocht, agus Dioscúrsa na mBan'. In: Combar 51 (5): 160-167. Nic Eoin, M. (1998) B'ait Leo Bean: Gnéithe den Idéeolaiocht Inscne i dTraidisiuin Liteartha na Gaeilge. Baile Átha Cliath: An Clóchomhar.

Nic Eoin, M. (2000) “Scéal ar an nGrá...” Na hAmhráin Ghrá agus an Smacht Sóisialta'. In: Riggs, P., Ó Conchúir, B. agus Ó Coileáin, S. (eag.) Saoi na hÉigse: Aistí in Ómós do Sheán Ó Tuama. Baile Átha Cliath: An Clóchomhar: 233-259.

Nietzsche, F.W. (1988 [1954]) 'From On Truth and Lie in an Extra-Moral Sense'. In W. Kaufmann (eag.) The Portable Nietzsche. Harmondsworth: Penguin Books: 42-47.

Ní Shíocháin, T. (2012) Bláth 's Craobh na nÚdar: Ambráin Mháire Bhuí. Baile Átha Cliath: Coiscéim.

Ní Shíocháin, T. (2018) Singing Ideas: Performance, Politics and Oral Poetry. New York \& Oxford: Berghahn.

Ní Shúilleabháin, E. (1997) Cois Abhann na Séad: Ambráin ó Mhúscraí. Dlúthdhiosca CICD132. Indreabhán: Cló Iar-Chonnachta.

Ó Conghaile, M., Ó Tuairisg, L. agus Ó Ceannabháin, P. (eag.) (2012) Leabhar Mór na nAmbrán. Indreabhán: Cló Iar-Chonnacht.

Ó Cróinín, D. (2000) The Songs of Elizabeth Cronin. Baile Átha Cliath: Four Courts Press.

Ó hEochaidh, S., Ní Néill, M. agus Ó Catháin, S. (eag.) (1977) Siscéalta ó Thir Chonaill. Fairy Legends from Donegal. Baile Átha Cliath: Comhairle Bhéaloideas Éireann.

Ó hÓgáin, D. (1982) An File: Staidéar ar Osnádúrthacht na Filiochta sa Traidisiún Gaelach. Baile Átha Cliath: Oifig an tSoláthair.

Ó Laoire, L. (2002) Ar Chreag i Lár na Farraige: Ambráin agus Ambránaithe i dToraigh. Indreabhán: Cló Iar-Chonnachta. 
Ó Laoire, L. (2016) 'Leithscéal ag na Mná: an Greann agus an Inscne i Scéal le Bab Feiritéar'. In: American Journal of Irish Studies 13 (1): 18-54.

Ó Madagáin, B. (1989) 'The Gaelic Lullaby: A Charm to Protect the Baby?'. In: Scottish Studies 29: 29-36.

Ó Madagáin, B. (1992) 'Echoes of Magic in the Gaelic Song Tradition'. In: Byrne, C.J., Harry, M. agus Ó Siadhail, P. (eag.) Celtic Languages and Celtic Peoples: Proceedings of the Second North American Congress of Celtic Studies, held in Halifax, August 16-19, 1989. Halifax Nova Scotia: D'Arcy McGee Chair of Irish Studies, Saint Mary's University: 125-140.

Ó Riain, P. (1971-1972) 'A Study of the Legend of the Wild Man'. In: Éigse 14: 179-206.

Partridge, A. (1980) 'Wild Men and Wailing Women'. In: Éigse 17: 25-37.

Petrie, G. (2002 [1855]) The Petrie Collection of the Ancient Music of Ireland (in eagar ag David Cooper agus Lillis Ó Laoire). Corcaigh: Cló Ollscoile Chorcaí.

Thomassen, B. (2014) Liminality and the Modern: Living Through the In-between. Farnham: Ashgate. Turner, V.W. (1969) The Ritual Process: Structure and Anti-Structure. London: Routledge.

Turner, V.W. (1985) On the Edge of the Bush. Tucson: University of Arizona Press.

Van Gennep, A. (1960) The Rites of Passage. Chicago: University of Chicago Press. 